\title{
Erratum
}

\section{Directed Projection Functions of Convex Bodies (Monatsh. Math. 149, 43-64 (2006))}

By

\author{
Paul Goodey ${ }^{1, *}$ and Wolfgang Weil ${ }^{2, *}$ \\ ${ }^{1}$ University of Oklahoma, Norman, OK, USA \\ ${ }^{2}$ Universität Karlsruhe, Germany
}

Published online August 21, 2006 (C) Springer-Verlag 2006

A comparison of the definitions of $\bar{v}_{1, j}(K ; \cdot)$ and $M_{j}^{d}$ in the above article shows that

$$
M_{j}^{d} h(K ; \cdot)=\kappa_{j-1} \bar{v}_{1, j}(K ; \cdot) .
$$

The factor $\kappa_{j-1}$ is missing in line 5 of Section 3.2, and in the following formulas: the second line of formula (11), the left-hand side of formula (12), and the righthand side of the first displayed formula in the proof of Lemma 1.

In the discussion of the body $K_{s}$ (at the end of Section 3.2), $\bar{v}_{1,2}\left(K_{s} ; \cdot\right)$ has, therefore, to be replaced by $2 \bar{v}_{1,2}\left(K_{s} ; \cdot\right)$ ( since $\left.\kappa_{1}=2\right)$.

Finally, the relation between the operators $M_{j}^{d}$ and $A_{j}^{d}$ given in the proof of Theorem 7 simplifies to

$$
M_{j}^{d} f=A_{j}^{d} f,
$$

for even functions $f$. Here, besides the missing factor $\kappa_{j-1}$, it has to be observed that in the formula from [15] which express $A_{j}^{d} f$ in terms of Radon transforms the factor $\sigma_{j-1}$ has to be corrected to $\sigma_{j}$.

The fact that $M_{j}^{d}$ and $A_{j}^{d}$ agree on even functions may seem surprising. However, it has a geometric explanation arising from the connection of $A_{j}^{d}$ to a different kind of directed projection function which is studied in the paper

Goodey P, Weil W (2006) Determination of convex bodies by directed projection functions (submitted)

\footnotetext{
* The research of the first author was supported in part by NSF Grant DMS-9971202 and that of the second author by a grant from the Volkswagen Foundation.
} 